Keywords: Dissociative symptoms; Overreporting; Dissociative Experiences Scale; Cambridge Depersonalisation Scale; Structured Inventory of Malingered Symptomatology.

\title{
The overlap between dissociative symptoms and symptom over-reporting
}

\author{
Harald Merckelbach ${ }^{\mathrm{a}, \star}$ \\ Timo Giesbrecht ${ }^{a}$ \\ Dalena van Heugten-van der Kloet ${ }^{\mathrm{b}}$ \\ Joop de Jong ${ }^{c}$ \\ Thomas Meyera \\ Kim Rietmana \\ ${ }^{a}$ Forensic Psychology Section, Maastricht \\ University, Maastricht \\ ${ }^{b}$ Nuffield Department of Clinical \\ Neurosciences, John Radcliffe \\ Hospital Oxford \\ c Psychotrauma Section, Parnassia Bavo \\ Clinics, The Hague \\ THE NETHERLANDS \\ UNITED KINGDOM
}

\begin{abstract}
Background and Objectives: The potential link between dissociative symptoms and symptom over-reporting has been given little attention. In two student samples $(N$ 's $=139$ and 113) and a clinical sample $(N=21)$, we examined whether self-reported dissociative symptoms are related to symptom over-reporting.

Methods: We relied on different measures of dissociation and over-reporting. In the clinical sample, we looked at whether the well-established link between dissociative symptoms and sleep disturbances would survive if we corrected for symptom over-reporting.

Results: Dissociativity correlated with symptom over-reporting in the student samples, but not in the clinical sample. Correcting for over-reporting tendencies did not fundamentally alter the relationships between dissociative symptoms and sleep disturbances in the clinical sample.

Conclusions: Our results suggest that the overlap between symptom over-reporting and dissociativity is much more a problem in nonclinical than in clinical samples.
\end{abstract}




\section{Introduction}

Dissociative symptoms form a heterogeneous group of subjective phenomena. They include such diverse experiences as having difficulties retrieving autobiographical memories and feelings of being an outside observer of one's body ${ }^{1}$. There exists no objective biomarker of dissociative psychopathology; its assessment depends entirely on self-report instruments ${ }^{2}$. The Dissociative Experiences Scale (DES) is a widely-used tool for this purpose. Its items screen for a broad range of dissociative symptoms, such as absorption, amnesia, and identity disturbances ${ }^{3}$. Another frequently used self-report scale is the Cambridge Depersonalisation Scale (CDS) that was specifically designed to screen for the subjective manifestations of depersonalisation (e.g., out-of-body experiences and lack of agency feelings $)^{4}$.

Neither the CDS nor the DES contains validity scales. This omission makes these instruments susceptible to symptom exaggeration $^{5,6,7}$. Surprisingly, systematic research looking into this potential problem is virtually absent. One pilot study ${ }^{8}$ administered the DES and a measure of symptom over-reporting (i.e., the Structured Inventory of Malingered Symptomatology; SIMS $^{9}$ ) to a student sample and found dissociativity and over-reporting to be positively correlated ( $r=0.51$ between DES and SIMS scores). This is a first indication that endorsement of dissociative symptoms may go along with self-reports of implausible symptoms. Such over-reporting might be the result of lenient standards for endorsing unusual experiences or careless responding ${ }^{10}$.

We examined to what extent self-reported dissociativity and symptom over-reporting overlap in nonclinical and clinical participants. If such overlap would be substantial, it would raise the question whether previously reported concomitants of dissociativity (e.g., unusual sleep experiences such as hypnagogic hallucinations ${ }^{11}$ ) refer to reliable correlates of dissociation, or to artefacts of over-reporting. A robust link between dissociativity and over-reporting would imply that researchers and clinicians alike would be well advised to employ validity checks along with dissociative assessment measures. With these considerations in mind, we explored whether DES and CDS scores of undergraduates (samples 1 and 2) and those of psychiatric inpatients (sample 3 ) correlate significantly with endorsement of implausible symptoms.

\section{Methods}

\section{Participants}

Sample 1 consisted of 154 (119 women) psychology undergraduates, with a mean age of 19.7 years $(S D=1.71$; range: $18-31$ years). Sample 2 consisted of 115 undergraduates (81 women). Their mean age was 22.2 years ( $S D=3.51$; range: $17-35$ years). Sample 3 was composed of 22 inpatients (18 women) who were hospitalized at a psychiatric facility specialized in trauma treatment. Their mean age was 38.8 years $(S D=10.15$; range: 20-59). The most prevalent diagnostic categories were Post Traumatic Stress Disorder (10 x), Dissociative Disorder (7 x), Mood Disorder (7 x), and Borderline Personality Disorder $(5 \mathrm{x})$, with many patients exhibiting comorbid psychopathology. Most patients were on medication, with benzodiazepines (5 x), sleep medication $(3 \mathrm{x})$, and SSRI's $(9 \mathrm{x})$ being the most frequently prescribed. The study was approved by the standing ethical committee of the Faculty of Psychology and Neuroscience. Participants took part on a voluntary basis and after they had signed a consent form. 


\section{Questionnaires}

Sample 1 (undergraduates) and sample 3 (patients) completed the $\mathrm{DES}^{3}$, which contains 28 items that ask for the frequency of disturbances in memory, perception, and awareness. Respondents use 100-mm visual analogue scales (anchors: $0=$ not at all; 100 = very much) to indicate the percentage of time they experienced these dissociative phenomena. Scores are averaged across items to obtain a total DES score (range: 0-100), with higher DES scores indicating a higher frequency of dissociative experiences. Values above $20^{12}$ or $30^{13}$ are considered to be indicative of clinically relevant dissociation.

Following the approach of Simeon and coworkers ${ }^{14}$, we also calculated DES-T scores by averaging across eight DES items (items $3,5,7,8,12,13,22$, and 27) that are thought to reflect pathological forms of dissociation. Simeon et al. ${ }^{14}$ recommended a cut score of 13 for the DES-T to identify clinically significant levels of dissociation.

Sample 1 also completed the Symptom Over-reporting Index (SOI $)^{15}$, which consists of four items that address the following noncredible symptoms: "I have headaches that are so severe my feet hurt"; "The buzzing in my ears keeps switching from the left to the right"; "I notice that the colour of objects around me keeps shifting"; and "I find myself frequently blacking out when I sit down." In previous research, these items were found to be most effective in separating instructed malingerers from honest responding participants ${ }^{16}$. Note also that several studies have demonstrated that even a small set of well-chosen validity items can distinguish careful from careless or exaggerating respondents ${ }^{10,15,17}$. The non-credible symptom items of the SOI were rated in keeping with the DES response format, i.e., on 100-mm visual analogue scales (anchors: $0=$ not at all; $100=$ very much). To obtain a mean SOI value, we averaged scores across the four items (range: 0-100).

Sample 2 (undergraduates) and sample 3 (patients) completed the $\mathrm{CDS}^{4}$, which consists of 29 symptoms related to depersonalisation and derealisation. Each symptom is rated on frequency (score 0-4) and duration (1-6). Frequency and duration scores are summed to obtain a total CDS score (range: 0-290), with higher scores indicating more frequent and/or more intense depersonalisation/derealisation symptoms. As recommended by Sierra and Berrios ${ }^{4}$, a cutoff of 70 was employed to identify respondents with clinically raised CDS scores.

Sample 2 (undergraduates) and sample 3 (patients) were also administered the SIMS ${ }^{9}$, which consists of 75 true-false items that describe atypical symptoms in several domains, among which psychosis, affective disorders, and low intelligence (e.g., "I have noticed that my body changes shape even though my weight stays the same"). Affirmative answers are summed to obtain a total SIMS score, with higher scores reflecting more symptom exaggeration.

Furthermore, sample 3 (patients) completed the Iowa Sleep Experience Survey (ISES) $)^{11,18}$, which lists 17 unusual sleep experiences that respondents rate on a 7 pointLikert scale (anchors: $1=$ never; $7=$ several times a week). Scores are summed, with higher total ISES scores indicating more selfreported sleep aberrations (e.g., waking dreams, sleep paralysis).

\section{Procedure and analysis}

After they had given informed consent, sample 1 was administered the DES and the SOI during a number of mass testing sessions. There were several orders in which the questionnaires were presented and these 
orders were counterbalanced over test sessions, although SOI items were always given after participants had completed the DES. Sample 2 was a convenience sample. That is, students who participated in four separate experiments unrelated to dissociative symptomatology were asked whether they were willing to complete two additional questionnaires, namely the CDS and the SIMS. The order in which the students were given the questionnaires was counterbalanced as much as possible. Patients in sample 3 participated in a sleep hygiene program. At the start of the program, they filled out the DES, CDS, SIMS, and ISES. Measures were given in a fixed order and patients had as much time as they needed to complete the scales.

To evaluate internal consistencies of the measures, Cronbach's $\alpha$ values were calculated. Pearson product-moment correlations were computed to examine the overlap between dissociation measures and over-reporting screens in each sample, separately. In sample 3 (patients), we tested with partial correlations whether the link between dissociative symptoms and sleep disturbances would remain intact if we would correct for the influence of over-reporting.

\section{Results}

Because of incomplete data, 15 participants were excluded from sample 1, two were excluded from sample 2, and one patient was removed from sample 3. Table 1 gives descriptive statistics for the samples. As can be seen, Cronbach's $\alpha$ 's for DES, CDS, and ISES were all in the 0.80-0.98 range. The SIMS attained an adequate alpha in sample 3, but alpha's for SOI and SIMS in the student samples were low. These modest alpha levels might be due to the limited number of items (SOI) or the binary response format (SIMS) and do not necessarily imply psychometric shortcomings ${ }^{19}$.

Mean total DES and DES-T scores in sample 1 were similar to those previously reported for student samples ${ }^{20}$. Sixty-one participants $(44 \%)$ had a total DES score $>20$, 32 participants $(23 \%)$ had a total DES score $>30$, and $50(36 \%)$ participants had a DES$\mathrm{T}$ score $>13$. The overall CDS score in sample 2 was in accordance with what is typically found in student samples ${ }^{21}$. Six students (5\%) scored above the clinical cutoff of the CDS.

DES and CDS were even more raised in the clinical sample: $90 \%$ had a total DES score $>20,71 \%$ had a total DES score $>30$, and $81 \%$ had a total DES-T score $>13$. Likewise, $81 \%$ of the patients had a CDS score that exceeded the cut score.

Table 1 also gives the Pearson product-moment correlations between dissociation measures and over-reporting screens. As can be seen, for the student samples, these correlations were significant and all in the mediumto-large effect size range (i.e., $r$ 's $=0.40-0.55$ ). However, for the clinical sample, these correlations were lower (i.e., $r$ 's $=0.25-0.32$ ) and fell short of significance.

In the clinical sample, sleep disturbances, as indexed by the ISES, were significantly related to the DES $(r=0.59, p<0.01)$, but not to the CDS $(r=0.40, p=0.07)$. Also, the association between SIMS and ISES fell short of significance ( $r=0.38, p=0.09$ ). Most importantly, the correlations between dissociation and sleep disturbances did not change drastically when the influence of SIMS was controlled for: The partial correlation between DES and ISES was $r=0.54, p=0.01$, and that between CDS and ISES was $r=0.31, p=0.18$. 
Table 1

Cronbach's alpha's of measures, mean scores (SD) of samples 1-3, and bivariate correlations [95\% CI].

\begin{tabular}{llll} 
& Cronbach's alpha & Mean $(\mathrm{SD})$ & $r[95 \% \mathrm{CI}]$ \\
\hline Sample 1 $(N=139)$ & & & $r(\mathrm{SOI}, \mathrm{DES})$ \\
DES Total & 0.94 & $21.0(13.9)$ & $0.55^{*}[0.42,0.66]$ \\
DES-T & 0.81 & $13.1(12.9)$ & $0.56^{*}[0.43,0.66]$ \\
SOI & 0.61 & $22.7(24.9)$ & - \\
\hline Sample 2 $(N=113)$ & & & $r(\mathrm{SIMS}, \mathrm{CDS})$ \\
Total CDS & 0.93 & $25.8(24.8)$ & $0.45^{*}[0.29,0.59]$ \\
CDS frequency & 0.90 & $9.0(7.7)$ & $0.44^{*}[0.28,0.58]$ \\
CDS duration & 0.95 & $16.8(19.3)$ & $0.40^{*}[0.23,0.54]$ \\
SIMS & 0.57 & $4.8(2.9)$ & - \\
\hline Sample 3 $(N=21)$ & & & $r(\mathrm{SIMS}, \mathrm{DES} / \mathrm{CDS} / \mathrm{ISES})$ \\
Total DES & 0.97 & $47.1(22.1)$ & $0.25[-0.24,0.65]$ \\
DES-T & 0.90 & $42.1(25.2)$ & $0.28[-0.19,0.65]$ \\
Total CDS & 0.98 & $127.9(52.6)$ & $0.32[-0.11,0.70]$ \\
CDS frequency & 0.96 & $62.3(27.1)$ & $0.33[-0.09,0.70]$ \\
CDS duration & 0.95 & $65.6(26.6)$ & $0.30[-0.18,0.70]$ \\
ISES & 0.81 & $73.5(15.6)$ & $0.38[-0.04,0.71]$ \\
SIMS & 0.85 & $25.2(8.6)$ & - \\
\hline
\end{tabular}

DES $=$ Dissociative Experiences Scale, $\mathrm{SOI}=$ Symptom Over-reporting Index, CDS = Cambridge Depersonalisation Scale; SIMS = Structural Inventory of Malingered Symptomatology; ISES = Iowa Sleep Experiences Scale. $* p<0.01$.

\section{Discussion}

We found that in student samples, dissociative experiences overlap with reports of non-credible symptoms. This connection may be carried by a subgroup of students who endorse low base-rate alternatives because they are bored by the test situation or adopt lenient response criteria. These respondents may inflate prevalence rates of dissociative symptoms and might be misclassified as clinical cases ${ }^{10}$. Indeed, in sample 1 , we observed high percentages of students (i.e., 23-44\%) scoring above the DES and DES-T cutoffs that are in use. In fact, these rates are implausibly high when one compares them to the prevalence of clinically significant disso- ciative symptoms in the general population, which have been estimated to be in the 5$29 \%$ range $^{13}$. The percentage of sample 2 students who exceeded the cutoff on the CDS was considerably lower (i.e., 5\%), but still well above prevalence rates of Depersonalisation Disorder, the most common dissociative disorder, which epidemiological studies estimate at $1.9 \%$ in the general population ${ }^{22}$.

Our results caution against the overly optimistic reliance on students' self-reports of dissociative symptoms. The practice to measure dissociative symptoms in nonclinical groups and relate these symptoms to other parameters without adjusting for over-reporting may account for contradictory findings. For example, some studies have reported that 
dissociative undergraduates have superior working memory capacity ${ }^{23}$, whereas others failed to replicate this ${ }^{24}$. The cognitive profile of dissociative individuals may become much clearer and better replicable, once researchers check for over-reporting and rule out respondents who manifest this tendency.

We might note that the phenomenon of symptom over-reporting is interesting in its own right. Careless responding, random responding, fatigue, and lack of effort are all known to operate in student samples and contribute to inflated symptom scores ${ }^{10,25,26}$. A clinically more interesting route to over-reporting is suggested by studies that found dissociation to be related to trait-like phenomena such as negative affectivity, alexithymia, and poor symptom perception ${ }^{27,28}$. Specifically, there might exist a subgroup of dissociative people whose primary problem is their difficulty in identifying somatic experiences. Lack of accurate interoceptive symptom perception, in turn, might fuel symptom over-reporting. Clearly, this route warrants further study.

Over-reporting of symptoms was also evident in our clinical sample, but here over-reporting was not reliably associated with dissociative symptomatology per se. As well, correcting for over-reporting did not drastically change the significant link between DES and self-reported sleep disturbances, indicating that these disturbances are a solid concomitant of broadly measured dissociative symptomatology $8,11,18$. The correlation between self-reported sleep disturbances and the more restricted category of depersonalisation/derealisation symptoms was less convincing and correcting for over-reporting did not change this pattern.

Several limitations of the current study deserve some comments. First, the SOI that we employed in sample 1 as an index of over-reporting consists of only four items. Although over-reporting can be detected with a rela- tively small set of items ${ }^{10,15,17}$, such brief screens are not able to shed light on the type of over-reporting. That is, individuals might respond randomly or carelessly to test items because they are annoyed by the test situation, but other respondents might engage in deliberate over-reporting (i.e., feigning) or symptom overendorsement on the basis of traits such as negative affectivity and alexithymia. Even more extensive scales, such as the SIMS $^{9}$ that we used in samples 2 and 3, are not able to differentiate between these various manifestations of over-reporting. Future studies would benefit from measures that discriminate between different pathways to over-reporting so as to develop articulated ideas about the origins of over-reporting in the context of dissociative symptoms.

Another limitation concerns our clinical sample (i.e., sample 3): It was highly heterogeneous in terms of comorbid symptoms and medication. Thus, our failure to detect a robust connection between dissociative symptoms and over-reporting might have to do with the noise introduced by comorbidity and medication. As well, our clinical sample was small, and future research testing the effects of adjustment for over-reporting in larger clinical samples is warranted. A crucial next step would be to test whether criterionrelated validity (e.g., dissociation predicting poor therapy outcome ${ }^{29}$ ) increases when symptom reports are adjusted for over-reporting tendencies.

To summarize, in student samples, but not in clinical samples, dissociative experiences are related to symptom over-reporting. Unless one assumes that items such as "I have headaches that are so severe my feet hurt" and "I have noticed that my body changes shape even though my weight stays the same" allude to authentic dissociative symptomatology, it is advisable to adjust DES and CDS data of nonclinical participants for the 
tendency to over-report symptoms. Excluding those who over-report symptoms might considerably deflate estimates of clinically relevant dissociation and may enhance generalizability to the clinical domain.

\section{References}

1. Holmes EA, Brown RJ, Mansell W, Fearon RP, Hunter EC, Frasquilho F, et al. Are there two qualitatively distinct forms of dissociation? A review and some clinical implications. Clin Psychol Rev. 2005; 25(1): 1-23.

2. Jureidini J. Does dissociation offer a useful explanation for psychopathology? Psychopathology. 2004; 37(6): 259-65.

3. Bernstein E, Putnam FW. Development, reliability and validity of a dissociation scale. J Nerv Ment Dis. 1986; 174(12): 727-35.

4. Sierra M, Berrios GE. The Cambridge Depersonalisation Scale: A new instrument for the measurement of depersonalisation. Psychiat Res. 2000; 93: 153-64.

5. Gilbertson A, Torem M, Cohen R, Newman I, Radojicic C, Patel S. Susceptibility of common self-report measures of dissociation to malingering. Dissociation. 1992; 5 : 216-20.

6. Candel I, Merckelbach H. Peritraumatic dissociation as a predictor of post-traumatic stress disorder: A critical review. Compr Psychiatry. 2004; 45(1): 44-50.

7. Merten T, Merckelbach H. Symptom validity testing in somatoform and dissociative disorders: A critical review. Psychol Inj and Law. 2013; 6: 122-37.

8. Giesbrecht T, Merckelbach H. Dissociatieve symptomen en slaap [Dissociative symptoms and sleep]. Tijdschr voor Psychiatrie. 2006; 48(3): 207-15.

9. Merckelbach H, Smith GP. Diagnostic accuracy of the Structured Inventory of Malingered Symptomatology (SIMS) in detecting instructed malingering. Arch Clin Neuropsychol. 2003; 18(2): 145-52.

10. Meyer JF, Faust KA, Faust D, Baker AM, Cook NE. Careless and random responding on clinical and research measures in the addictions: A concerning problem and investigation of their detection. Int J Ment Health Addiction. 2013; 11: 292-306.

11. van der Kloet D, Merckelbach H, Giesbrecht T, Lynn SJ. Fragmented Sleep, Fragmented Mind: The role of sleep in Dissociative Symptoms. Perspect Psychol Sci. 2012; 7(2): 159-75.
12. Mueller-Pfeiffer C, Rufibach K, Wyss D, Perron N, Pitman RK, Rufer M. Screening for dissociative disorders in psychiatric out- and day care-patients. J Psychopathol Behav Asses. 2013; 35: 592-602.

13. Putnam FW, Carlson EB, Ross CA, Anderson G, Clark $\mathrm{P}$, Torem M, et al. Patterns of dissociation in clinical and nonclinical samples. J Nerv Ment Dis. 1996; 184(11): 673-9.

14. Simeon D, Guralnik O, Gross S, Stein D, Schmeidler J, Hollander E. The detection and measurement of depersonalization disorder. J Nerv Ment Dis. 1998; 186(9): 536-42.

15. Merckelbach H, Langeland W, de Vries G, Draijer N. Symptom overreporting obscures the dose-response relationship between trauma severity and symptoms. Psychiat Res. 2014; 217(3): 215-9.

16. Merckelbach H, Smeets T, Jelicic M. Onwaarschijnlijke symptomen simuleren: de Wildman Symptom Checklist: De Wildman Symptom Checklist. Neuropraxis. 2008; 12: 53-7.

17. Cooper DB, Nelson L, Armistead-Jehle P, Bowles AO. 2011. Utility of the Mild Brain Injury Atypical Symptom Scale as a screening measure for symptom over-reporting in Operation Enduring Freedom/Operation Iraqi Freedom service members with post-concussive complaints. Arch Clin Neuropsychol. 2011; 26(8): 718-27.

18. Watson D. Dissociation of the night: Individual differences in sleep-related experiences and their relation to dissociation and schizotypy. J Abnorm Psychol. 2001; 110(4): 526-35.

19. Schmitt N. Uses and abuses of coefficient alpha. Psychol Assessment. 1996; 8: 350-3.

20. Giesbrecht T, Merckelbach H, Gerarts E. The dissociative experiences taxon is related to fantasy proneness. $\mathrm{J}$ Nerv Ment Dis. 2007; 195(9): 769-72.

21. Aardema F, Wu KD. Imaginative, dissociative, and schizotypal processes in obsessive-compulsive symptoms. J Clin Psychol. 2011; 67(1): 74-81.

22. Michal M, Wiltink J, Subic-Wrana C, Zwerenz R, Tuin I, Lichy M, et al. Prevalence, correlates, and predictors of depersonalization experiences in the German general population. J Nerv Ment Dis. 2009. 197(7): 499-506.

23. Veltman DJ, de Ruiter MB, Rombouts SA, Lazeron RH, Barkhof F, Van Dyck R, et al. Neurophysiological correlates of increased verbal working memory in high-dissociative participants: A functional MRI study. Psychol Med. 2005; 35(2): 175-85.

24. Wright DB, Osborne JE. Dissociation, cognitive failures, and working memory. Am J Psychol. 2005; 118(1): 103-13. 
25. De Jonge P, Slaets JPJ. Response set in self-report data and their associations with personality traits. Eur J Psychiat. 2005; 19(4), 209-14.

26. An KY, Zakzanis KK, Joordens S. Conducting research with non-clinical healthy undergraduates: Does effort play a role in neuropsychological test performance? Arch Clin Neuropsychol. 2012; 27(8): 849-57.

27. Elzinga B, Bermond B, van Dyck R. The relationship between dissociative proneness and alexithymia. Psychother Psychosom. 2002; 71(2): 104-11.

28. Bogaerts K, Millen A, Li W, De Peuter S, Van Diest I, Vlemincx, et al. High symptom reporters are less interoceptively accurate in a symptom-related context. J Psychosom Res. 2008; 65(5): 417-24.
29. Kleindienst N, Limberger MF, Ebner-Priemer UW, Keibel-Mauchnik J, Dyer A, Berger M, et al. Dissociation predicts poor response to Dialectial Behavioral Therapy in female patients with Borderline Personality Disorder. Journal Personal Disord. 2011; 25(4): 432-47.

* Corresponding author:

Prof. Dr. Harald Merckelbach

Forensic Psychology Section

Faculty of Psychology and Neuroscience

Maastricht University, PO Box 616

6200 MD, Maastricht

The Netherlands

E-mail: H.Merckelbach@maastrichtuniversity.nl 